\title{
Analysis of ALTAIR 1998 Meteor Radar Data
}

\author{
J. Zinn • S. Close • P.L. Colestock • A. MacDonell • R. Loveland
}

\begin{abstract}
We describe a new analysis of a set of 32 UHF meteor radar traces recorded with the 422 MHz ALTAIR radar facility in November 1998. Emphasis is on the velocity measurements, and on inferences that can be drawn from them regarding the meteor masses and mass densities. We find that the velocity vs altitude data can be fitted as quadratic functions of the path integrals of the atmospheric densities vs distance, and deceleration rates derived from those fits all show the expected behavior of increasing with decreasing altitude. We also describe a computer model of the coupled processes of collisional heating, radiative cooling, evaporative cooling and ablation, and deceleration - for meteors composed of defined mixtures of mineral constituents. For each of the cases in the data set we ran the model starting with the measured initial velocity and trajectory inclination, and with various trial values of the quantity $\mathrm{mos}_{\mathrm{s}}{ }^{2}$ (the initial mass times the mass density squared), and then compared the computed deceleration vs altitude curves vs the measured ones. In this way we arrived at the best-fit values of the $\mathrm{m} \rho_{\mathrm{s}}{ }^{2}$ for each of the measured meteor traces. Then further, assuming various trial values of the density $\rho_{\mathrm{s}}$, we compared the computed mass vs altitude curves with similar curves for the same set of meteors determined previously from the measured radar cross sections and an electrostatic scattering model. In this way we arrived at estimates of the best-fit mass densities $\rho_{\mathrm{s}}$ for each of the cases.
\end{abstract}

Keywords meteor $\cdot$ ALTAIR $\cdot$ radar analysis

\section{Introduction}

This paper describes a new analysis of a set of $422 \mathrm{MHz}$ meteor scatter radar data recorded with the ALTAIR High-Power-Large-Aperture radar facility at Kwajalein Atoll on 18 November 1998. The exceptional accuracy/precision of the ALTAIR tracking data allow us to determine quite accurate meteor trajectories, velocities and deceleration rates. The measurements and velocity/deceleration data analysis are described in Sections II and III. The main point of this paper is to use these deceleration rate data, together with results from a computer model, to determine values of the quantities $m \rho_{s}{ }^{2}$ (the meteor mass times its material density squared); and further, by combining these $m \rho_{\mathrm{s}}{ }^{2}$ values with meteor mass estimates for the same set of meteors determined separately from measured radar scattering cross sections, to arrive at estimates of the mass densities $\rho_{\mathrm{s}}$.

The computer model, described in Section IV and Appendix A, treats the simultaneous processes of meteor heating through air molecule collisions, blackbody radiation emission, evaporation, sputtering,

\footnotetext{
J. Zinn $(\varangle) \cdot$ P. L. Colestock $\bullet$ R. Loveland

Los Alamos National Laboratory, Los Alamos, NM. E-mail: jzinn@lanl.gov

S. Close

Stanford University, Stanford, CA

A. MacDonell

Boston University, Boston, MA
} 
and deceleration - for meteors of specified assumed initial mixtures of mineral constituents. The model assumes in each case that the meteors are spherical, and remain so without fragmenting. It includes an imbedded table of atmospheric mass densities vs altitude, and data on (1) vapor pressure vs temperature, (2) heat of sublimation, (3) vapor molecular weight, and (4) melting point - for each of the assumed constituent species. Other inputs to the model include, for each individual case, (1) the initial meteor velocity and trajectory inclination (i.e. at the top of the atmosphere), (2) trial values of the initial $m \rho_{\mathrm{s}}{ }^{2}$ (i.e. values before entering the atmosphere).

The data include 32 individual meteor traces, where the meteors all appear to be in the mass range $10^{-6}$ to $10^{-4}$ grams, and the altitudes are such that air molecule collision mean free paths are much larger than the meteor dimensions. Thus air molecule collisions with the meteor can be regarded as isolated events, and fluid-dynamic effects do not apply (large Knudsen number). In our data analysis we fit the reduced data on velocities vs altitude and trajectory inclination as least-squares quadratic functions of the path-integrated air column densities, using tabular data on air densities vs altitude. We then compute the corresponding deceleration rates. We find, as expected, that for all the traces the deceleration rates increase with decreasing altitude.

The model equations and variables are listed in Appendix A. Appendix B describes a quasianalytic solution of the ablation equations for a 1-component meteor, using the steady-state approximation. It shows that at the lowest altitudes the meteor temperatures are determined mainly by an equilibrium between collisional heating and evaporative cooling. And the ablation coefficients tend to approach a common value equal to the vapor molecular weight divided by twice the heat of vaporization, and independent of the initial meteor velocity.

\section{Experimental}

The ALTAIR High-Power-Large-Aperture radar facility is located on the Kwajalein Atoll $\left(9^{\circ} \mathrm{N}, 167^{\circ}\right.$ E) in the Republic of the Marshall Islands. ALTAIR has a 43-m diameter mechanically-steered parabolic dish, and simultaneously transmits a peak power of $6 \mathrm{MW}$ at two frequencies (VHF-160 MHz, and UHF-422 MHz). (Close et al 2000, Close et al 2004 ). The radar characteristics are described in detail in those references. It is particularly suited for precise measurements of small targets at long ranges. Extensive measurements going back to 1983 show stable rms tracking accuracies of \pm 15 millidegrees in angle and $\pm 6 \mathrm{~m}$ in range. In the present paper we discuss a UHF data set consisting of 32 meteor traces obtained on November 18, 1998. The radar sample window encompassed slant ranges corresponding to heights mostly between 90 to $110 \mathrm{~km}$. $150 \mu$ s pulsed waveforms were used, with a range sample spacing corresponding to about 7.5 meters. The instantaneous meteor 3-dimensional positions were determined from the monopulse range and angular measurements, and the velocities were determined by direct numerical differencing of the positions vs time (Close et al, 2002).

In this paper we do not yet make use of a much larger set of ALTAIR meteor data obtained in 2007 and 2008, or results of an ongoing analysis of these data where line-of-sight velocities are determined from measured Doppler frequency shifts of the reflected radar signals (Loveland et al, 2010). We expect that the velocities thus determined will be of higher accuracy than those derived from the 1998 data described in this paper. We will report analyses of the newer results in a later paper. 


\section{Data Analysis}

For each of the 32 meteor traces (using the tabulated altitudes, velocities and vertical velocity components vs time) we begin by performing a quadratic least-squares fit to the velocities vs the air path traversed (Q), where

$$
\mathrm{Q} \equiv \int_{\mathrm{z}}^{\infty} \rho \mathrm{ds}
$$

$\rho$ is the local air density, and ds is the element of distance along the meteor path to the altitude $\mathrm{z}$. The $\rho$ 's were taken from the CIRA '61 tabulations (COSPAR International Reference Atmosphere 1961), and the Q integrals were evaluated for each point along each trace using the measured trajectory inclination angles. (We will regard these atmospheric density data as given, and note that they are probably more accurately determined than are the meteor masses or mass densities that we will derive from the radar data). Then from the $\mathrm{Q}$ derivative of this fitted quadratic velocity vs $\mathrm{Q}$ function we compute the corresponding deceleration rates as functions of $\mathrm{z}$. Figure 1 is a composite plot of the fitted velocities vs altitude for the 32 traces. It will be noted that they all show velocities decreasing with decreasing altitude, and all of them show some downward curvature. Likewise, the deceleration rates increase with decreasing altitude, as they should. Figure 2 is a composite plot of the decelerations (negative accelerations) vs altitude for the 32 traces, derived from the velocity fits. (Note that one meteor streak appears to be interstellar in origin, with a velocity exceeding $72.8 \mathrm{~km} / \mathrm{s}$. We will perform orbital analysis on this streak in the future to confirm this result.) We note also that in five of the cases the initial value of $\mathrm{dv} / \mathrm{dt}$ has come out to be positive, due presumably to inaccuracies in the velocity data. These cases will be discarded as flawed.

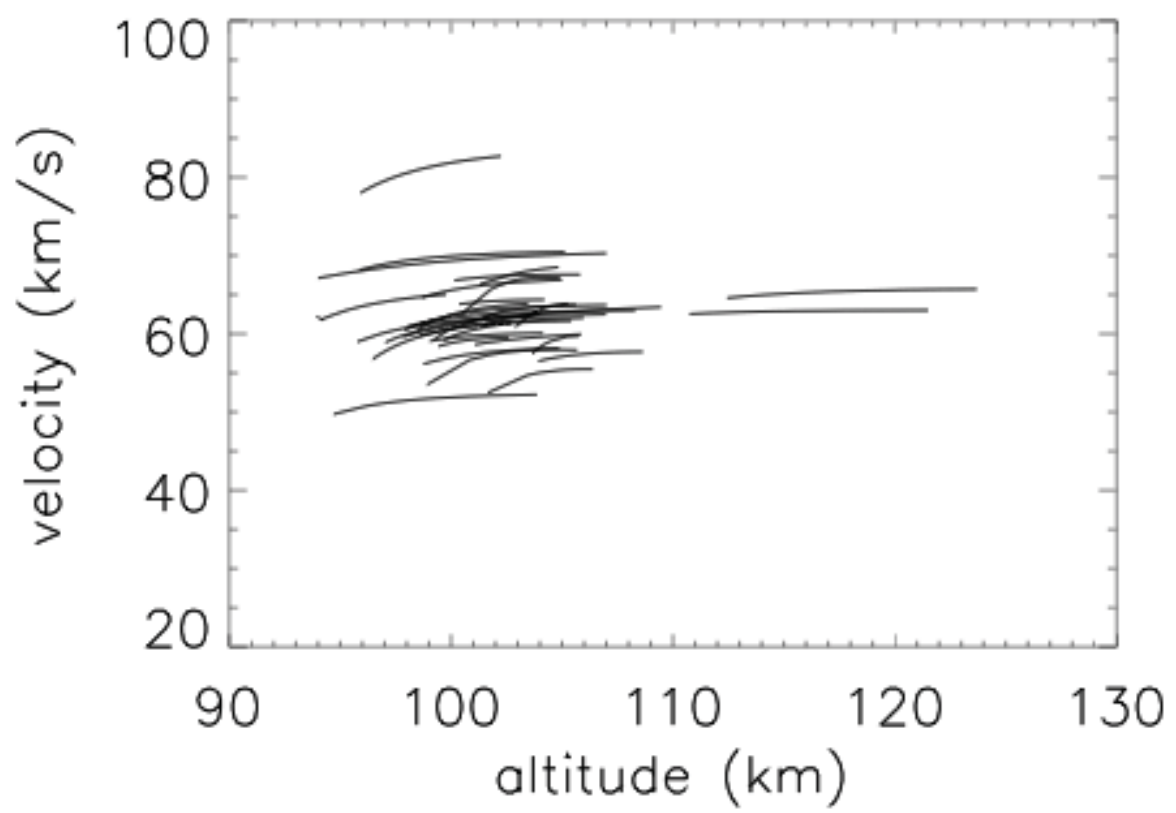

Figure 1. Composite plot of the fitted velocities vs altitude for the 32 cases - from the least-squares quadratic fits of the measured velocities vs Q. 


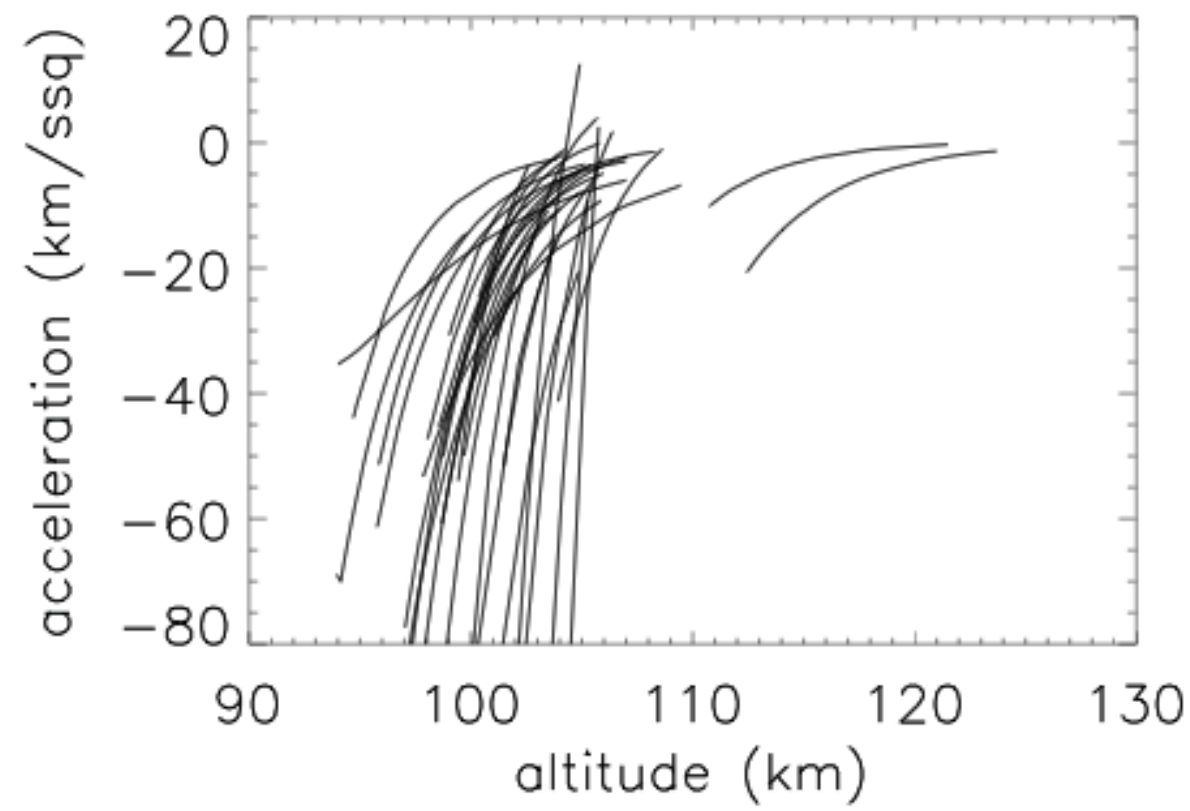

Figure 2. Composite plot of the accelerations vs altitude for the 32 cases - as derived from the quadratic fits to the velocities

In our analysis we will assume that the meteors are spherical. Then the energy flux on the meteor surface due to air molecule collisions is $\pi r^{2} \rho v^{3} / 2$, where $r$ and $v$ are the meteor radius and velocity, and $\rho$ is the local air density. Consistent with other authors (e.g. Pecina \& Ceplecha, 1982; Opik, 1958; Bronshten, 1983) we will write the meteor mass loss rate as

$$
\mathrm{dm} / \mathrm{dt}=-\pi \mathrm{r}^{2} \rho v^{3} \sigma
$$

where $\mathrm{m}$ is the meteor mass and $\sigma$ is the "ablation coefficient". The rate of deceleration of the meteor is

$$
\mathrm{dv} / \mathrm{dt}=-\pi \mathrm{r}^{2} \rho \mathrm{v}^{2} / \mathrm{m}
$$

Combining equations 1 and 2 we obtain

$$
\mathrm{dm} / \mathrm{m}=\sigma \mathrm{vdv}
$$

If we make the convenient (but not necessarily valid) assumption that $\sigma$ is constant, then Eq 3 can be integrated, giving

$$
\ln \left(\mathrm{m} / \mathrm{m}_{1}\right)=(\sigma / 2)\left(\mathrm{v}^{2}-\mathrm{v}_{1}^{2}\right)
$$

where $\mathrm{m}_{1}$ and $\mathrm{v}_{1}$ are the initial values of $\mathrm{m}$ and $\mathrm{v}$ along a given meteor radar trace. The constant- $\sigma$ assumption would be appropriate if, for instance, the meteor mass loss was dominated by "sputtering".

In an alternative model (e.g. Vondrak et al, 2008; Janches et al, 2009; Lebedinets, 1973), the mass loss is dominated by thermal evaporation of the meteor constituents. The instantaneous 
evaporation rate is determined by the instantaneous temperature. In the next section we describe our own numerical model of these coupled processes.

\section{Numerical Model}

The consensus of most current theoretical studies of the ablation and slowing down of small meteors in the atmosphere (Lebedinets, 1973; Janches et al, 2009; Vondrak et al, 2008) is that: (1) Very rapid heating occurs due to collisions with air molecules, moderated by energy losses due to blackbody emission from the meteor surface and due to evaporation. (2) The heating leads to vaporization of meteor (generally preceded by melting). (3) Some sputtering occurs, in addition to the vaporization. (4) The air molecule collisions also lead to deceleration of the meteor. (5) With very small meteors the rate of internal heat conduction is sufficient to maintain a uniform temperature distribution within the meteor. (6) Meteors are composed of mixtures of chemical constituents, and each will vaporize at its own rate.

It the present model we further assume that the meteor is spherical, and that after melting it does not disintegrate.

The rate of heating of the meteor through air molecule collisions is $(\mathrm{dH} / \mathrm{dt})_{\text {coll }}=\pi \mathrm{r}^{2} \rho \mathrm{v}^{3} / 2$, where $\mathrm{r}$ is the instantaneous meteor radius, $\mathrm{v}$ its instantaneous velocity, and $\rho$ the local air density, and it is assumed that all the energy of a collision is transferred to the meteor. The rate of loss of energy by blackbody emission is $(\mathrm{dH} / \mathrm{dt})_{\mathrm{rad}}=-4 \pi \mathrm{r}^{2} \sigma_{\mathrm{SB}} \mathrm{T}^{4}$, where $\sigma_{\mathrm{SB}}$ is the Stephan-Boltzmann constant and $\mathrm{T}$ is the instantaneous temperature. The vapor pressure of the ith chemical constituent of the meteor is given by the Claussius-Clapeyron equation

$$
\mathrm{P}_{\mathrm{vap}(\mathrm{i})}=\mathrm{A}_{\mathrm{i}} \exp \left(-\mathrm{C}_{\mathrm{i}} / \mathrm{T}\right)
$$

where $A_{i}$ and $C_{i}$ are constants characteristic of the particular constituent. The evaporative flux of each constituent from the surface is given by the Langmuir relation

$$
\mathrm{F}_{\text {evap(i) }}=\mathrm{C}_{\mathrm{flx}} \mathrm{P}_{\mathrm{vap}(\mathrm{i})} /\left(\mu_{\mathrm{vap}(\mathrm{i})} \mathrm{T}\right)^{1 / 2} \quad\left(\text { molecules } / \mathrm{cm}^{2} \mathrm{~s}\right),
$$

(Taylor and Langmuir 1933), where $\mu_{\mathrm{vap}(\mathrm{i})}$ is the molecular weight of the vapor. . If $\mathrm{P}_{\mathrm{vap}(\mathrm{i})}$ is in dynes $/ \mathrm{cm}^{2}$ and $\mu_{\mathrm{vap}(\mathrm{i})}$ is in grams, then the constant $\mathrm{C}_{\mathrm{flx}}$ is equal to $3.40 \times 10^{7}$. Then the rate of energy loss from the meteor surface due to evaporation of each constituent is

$$
(\mathrm{dH} / \mathrm{dt})_{\text {evap }}=-4 \pi \mathrm{r}^{2} \Delta \mathrm{H}_{\mathrm{sblm}(\mathrm{i})} \mathrm{F}_{\text {evap(i) }}
$$

where $\Delta \mathrm{H}_{\mathrm{sblm}(\mathrm{i})} \quad$ is the heat of sublimation (erg/molecule). Then the rate of change of the meteor temperature is

$$
\mathrm{dT} / \mathrm{dt}=\left[(\mathrm{dH} / \mathrm{dt})_{\mathrm{coll}}+(\mathrm{dH} / \mathrm{dt})_{\mathrm{rad}}+\sum_{(\mathrm{i}=1, \mathrm{~N})}(\mathrm{dH} / \mathrm{dt})_{\mathrm{evap}(\mathrm{i})}\right] / \mathrm{C}_{\mathrm{p}},
$$

where $C_{p}$ is the specific heat. In the above equation it is assumed that each constituent vaporizes at a rate independent of the other constituents, as long as that constituent is still present (i.e. has not totally evaporated). Values of the parameters $\Delta \mathrm{H}_{\mathrm{sblm}(\mathrm{i})}, \mathrm{A}_{\mathrm{i}}, \mathrm{C}_{\mathrm{i}}, \mu_{\mathrm{vap}(\mathrm{i})}$ and melting point for several likely meteor constituents are listed in Table 1, below. 
Table 1. Physico-chemical parameters for meteor constituents, and references. The numbered references are: 1. Ferguson et al, 2004; 2. Brewer et al, 1948; 3. Clarke and Fox, 1969; 4. Wickramasinghe and Swamy, $1968 ; 5$. Brewer and Porter, 1954; 6. Akopov, 1999; 7.Fabian,1993; 8. Patnaik, 2002.

\begin{tabular}{|l|l|l|l|l|l|l|}
\hline Constituent & $\Delta \mathrm{H}_{\text {sblm }}$ & $\mathrm{A}$ & $\mathrm{C}$ & $\mu_{\text {vap }}$ & Melting Pt & References \\
\hline & $(\mathrm{erg} / \mathrm{molec})$ & dyne $/ \mathrm{cm}^{2}$ & $\mathrm{Deg} \mathrm{K}$ & grams & Deg K & \\
\hline & & & & & & \\
\hline Fe (iron metal) & $6.62 \mathrm{e}-12$ & $5.06 \mathrm{e}+12$ & $4.836 \mathrm{e}+4$ & $9.30 \mathrm{e}-23$ & 1811 & 1 \\
\hline $\mathrm{C}$ (graphite) & $1.495 \mathrm{e}-11$ & $9.74 \mathrm{e}+15$ & $1.006 \mathrm{e}+5$ & $3.99 \mathrm{e}-23$ & --- & 2,3 \\
\hline $\mathrm{SiO}_{2}$ & $9.64 \mathrm{e}-12$ & $4.16 \mathrm{e}+11$ & $6.99 \mathrm{e}+4$ & $9.97 \mathrm{e}-23$ & 1923 & 4 \\
\hline $\mathrm{MgO}$ & $8.65 \mathrm{e}-12$ & $9.16 \mathrm{e}+14$ & $6.27 \mathrm{e}+4$ & $6.64 \mathrm{e}-23$ & 3073 & 5 \\
\hline $\mathrm{FeO}$ & $1.03 \mathrm{e}-11$ & $1.01 \mathrm{e}+16$ & $7.47 \mathrm{e}+4$ & $1.20 \mathrm{e}-22$ & 1653 & $6,7,8$ \\
\hline
\end{tabular}

Table 1 shows the physico-chemical parameters that we have assumed for several possible meteor constituents, including $\mathrm{A}, \mathrm{C}, \Delta \mathrm{H}_{\mathrm{sblm}}$, and some references. In most cases the references do not give the quantities $\mathrm{A}, \mathrm{C}$ and $\Delta \mathrm{H}_{\text {sblm }}$ directly, and in those cases we have had to calculate those quantities by fitting Eq 6 to data on vapor pressures measured at two or more temperatures, and assuming that $\Delta \mathrm{H}_{\text {sblm }}$ is equal to the Boltzmann constant $\mathrm{k}_{\mathrm{B}}$ times $\mathrm{C}$. The references listed in Table 1 are mostly sources of vapor pressure and/or boiling point data.

The rate of loss of mass from the meteor due to evaporation, is

$$
(\mathrm{dm} / \mathrm{dt})_{\mathrm{evap}}=-4 \pi \mathrm{r}^{2} \sum_{(\mathrm{i}=1, \mathrm{~N})}\left(\mu_{\mathrm{vap}(\mathrm{i})} \mathrm{F}_{\text {evap(i) }}\right) \text {. }
$$

There will also be some mass loss due to sputtering, given by $(\mathrm{dm} / \mathrm{dt})_{\text {sputt }}=\pi \mathrm{r}^{2} \rho \mathrm{v}^{3} \sigma_{\text {sputt }}$, where $\sigma_{\text {sputt }}$ is the ablation coefficient associated with sputtering (units of $\mathrm{s}^{2} / \mathrm{cm}^{2}$ ). The meteor radius $\mathrm{r}$ is related to the mass $\mathrm{m}$ by $\mathrm{r}=\left(3 \mathrm{~m} / 4 \pi \rho_{\mathrm{s}}\right)^{1 / 3}$, where $\rho_{\mathrm{s}}$ is the mass density of the solid meteor.

Finally, the rate of deceleration of the meteor is given by Eq 3 .

$$
\mathrm{dv} / \mathrm{dt}=-\pi \mathrm{r}^{2} \rho v^{2} / \mathrm{m}
$$

We have developed our own computer model that incorporates the above processes in the form of a set of ordinary differential equations expressing the rates of change of meteor mass, velocity, radius, temperature, etc. as functions of time. The input meteor composition can be either a pure compound or a mixture of compounds. The differential equations are detailed in Appendix A. This model appears to be very similar to the one described by Vondrak et al, 2008.

Some key questions are, of course: (1) What is the meteor composition? (2) What is its mass density? (3) Does the meteor actually remain intact after it melts? and (4) What is the contribution of sputtering to the total ablation coefficient?

Figures 3a-3f show comparisons, for a set of six traces, of computed vs measured decelerations vs altitude. For inputs to the computations for each trace we take (1) the measured initial velocity; (2) the measured trajectory inclination angle; (3) an assumed initial value of $m \rho_{\mathrm{s}}{ }^{2}$, which is shown on the plot; (4) an assumed initial composition -- namely an equimolar mixture of $\mathrm{SiO}_{2}, \mathrm{FeO}$ and $\mathrm{MgO}$ (which corresponds roughly to the expected decomposition products of olivine, a mineral that is commonly found in stony meteorites); (5) an assumed energy for sputtering, $\mathrm{E}^{*}=15 \mathrm{eV}$ per molecule, giving a constant sputtering contribution of $2.1 \times 10^{-12} \mathrm{~s}^{2} / \mathrm{cm}^{2}$ to the total ablation coefficients; (6) a mass density 
$\rho_{\mathrm{s}}$ of $1.0 \mathrm{~g} / \mathrm{cm}^{3}$. With these assumptions Figures 3a-3f show good agreement between the computed results and the data. In all, we found satisfactory agreement in 20 of the 32 cases.
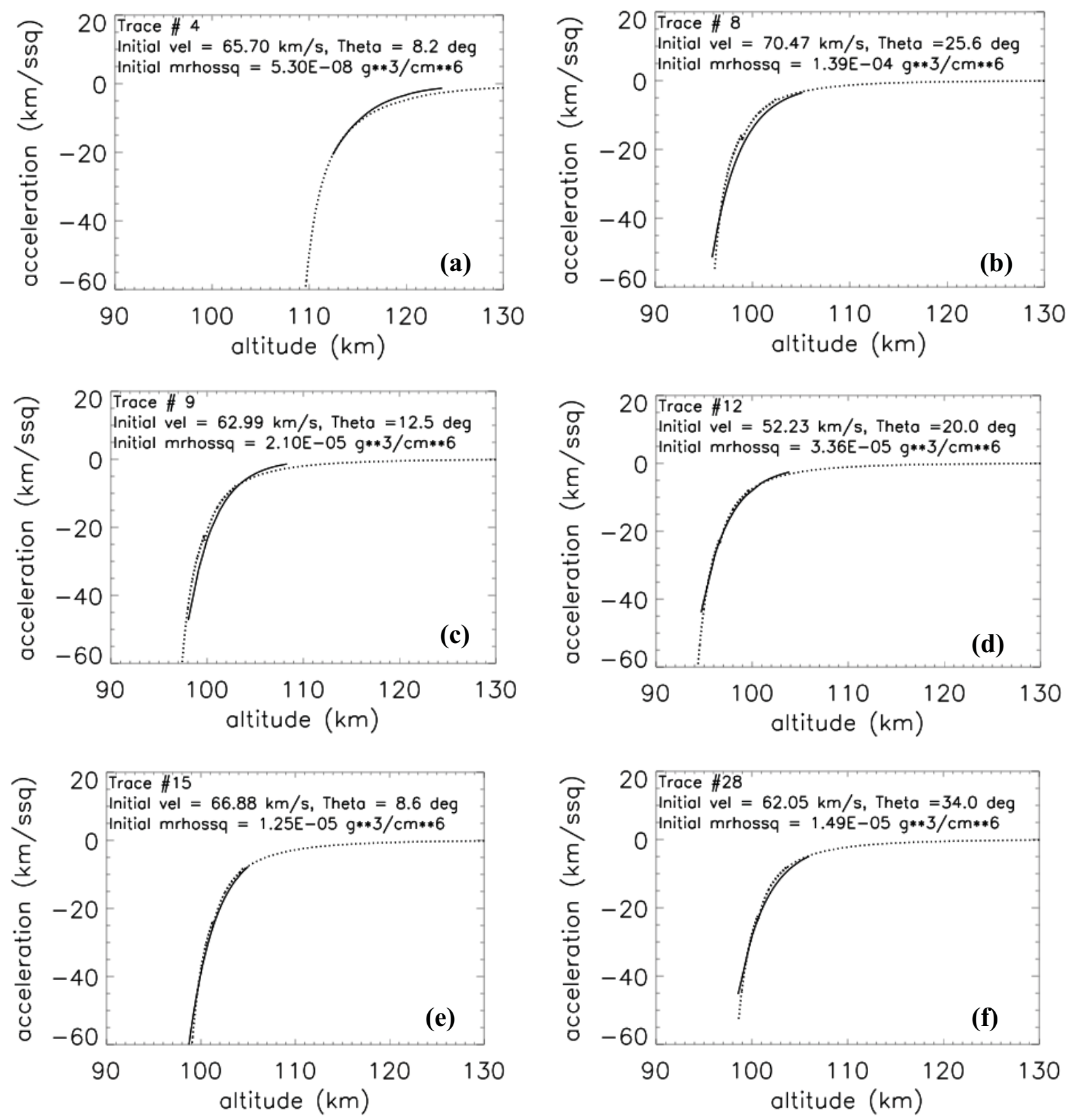

Figures 3a-3f. In each of these plots the solid curve is the acceleration derived from the data fit, and the dotted curve is the one computed with the model (with inputs described in the text).

We also ran computations with other assumed mass densities $\left(\rho_{\mathrm{s}}\right)$ and sputtering energies $\left(\mathrm{E}^{*}\right)$, although the results will not be shown here. From comparisons of the results with Figs $3 a-3 f$ we found that the computed deceleration rates did not depend at all on the assumed density $\rho_{\mathrm{s}}$. This is to be 
expected, since the initial meteor mass is equal to the input $m \rho_{\mathrm{s}}^{2}$ divided by $\rho_{\mathrm{s}}{ }^{2}$, and the separate dependencies of the acceleration rates on $m$ and $\rho_{\mathrm{s}}$ are always connected through the $m \rho_{\mathrm{s}}{ }^{2}$.

We also found that without the assumed relatively large sputtering contribution to the ablation coefficients the agreement with the data was less good than the extent of agreement shown in Figs 3a-3f.

Although the computed deceleration values are independent of the separate values of $\mathrm{m}$ and $\rho_{\mathrm{s}}$ (once the value of the product $m \rho_{\mathrm{s}}{ }^{2}$ is prescribed), the mass $\mathrm{m}$ is of course equal to $\mathrm{m} \rho_{\mathrm{s}}{ }^{2} / \rho_{\mathrm{s}}{ }^{2}$. Because of this it is possible to arrive at rough estimates of both $\mathrm{m}$ and $\rho_{\mathrm{s}}$ separately, using the measured values of decelerations and radar cross sections in combination, and using the Close et al electrostatic scattering model (Close et al, 2004) together with our present ablation and deceleration model. Figures $4 \mathrm{a}$ and $4 \mathrm{~b}$ show two examples of such attempts to determine both $\mathrm{m}$ and $\rho_{\mathrm{s}}$ from the experimentally determined $\mathrm{m} \rho_{\mathrm{s}}{ }^{2}$ and "mass1" (mass from the radar cross sections). In Figure 4a, representing trace \#8, the four solid curves are the computed inertial mass values vs altitude derived from the best-fit $\mathrm{m}_{\mathrm{s}}{ }^{2}$ (from Fig $3 \mathrm{~b}$ ) assuming four different values of $\rho_{\mathrm{s}}$, namely $0.1,0.316,1.0$ and $3.16 \mathrm{~g} / \mathrm{cm}^{3}$, while the dashed curve is mass 1 . In this case it appears that the best-fit density $\rho_{\mathrm{s}}$ is about $1 \mathrm{~g} / \mathrm{cm}^{3}$, and the initial pre-ablation mass is about $1 \times 10^{-4} \mathrm{~g}$. Figure $4 \mathrm{~b}$ is a similar set of plots, but representing trace $\# 9$ (from Fig $3 \mathrm{c}$ ). In this case the best-fit $\rho_{\mathrm{s}}$ is about 0.3 and the initial mass is again about $1 \times 10^{-4} \mathrm{~g}$. We have made similar plots (not shown here) for each of the other measured traces, and we find that the average best-fit $\rho_{\mathrm{s}}$ is about 0.5 , but with a spread of values between about 0.1 and 1 .
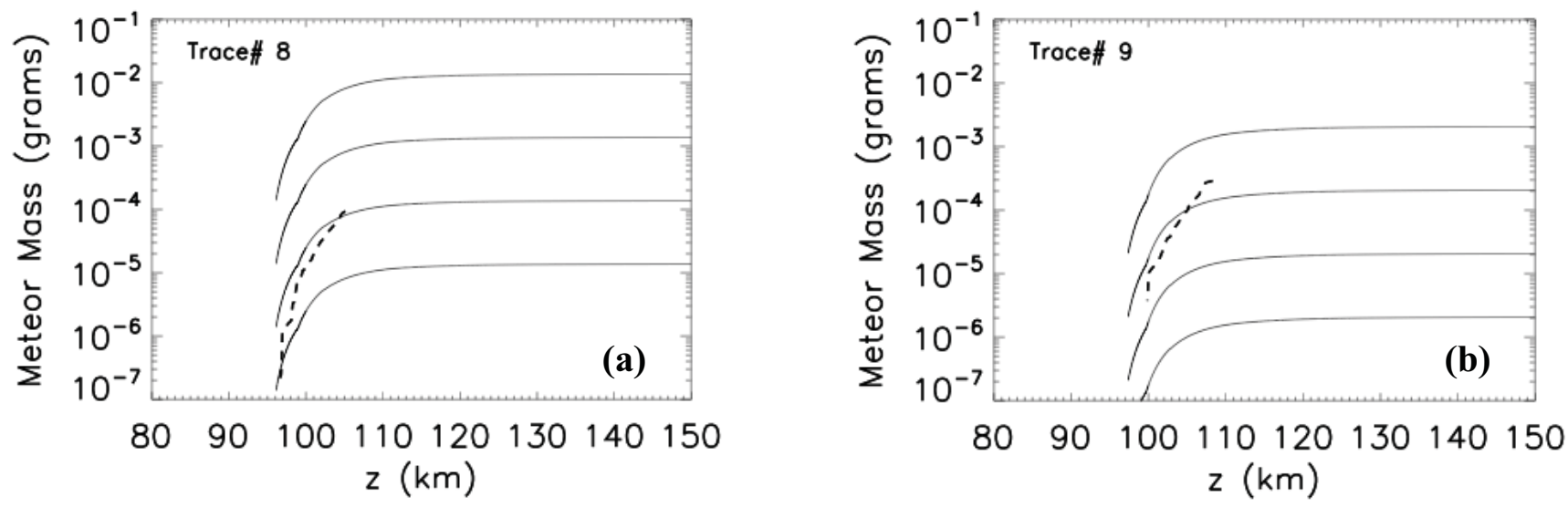

Figures 4. (a) Here the solid curves are the inertial masses (for trace \#8) computed with the numerical model, using the best-fit value of $m \rho_{\mathrm{s}}{ }^{2}$ together with four different assumed values of the meteor density $\rho_{\mathrm{s}}$, namely (from top to bottom) $0.1,0.316,1.0$ and $3.16 \mathrm{~g} / \mathrm{cm}^{3}$. The dashed curve is the "mass 1" (mass determined from the measured cross sections together with the electrostatic scattering model. (b) Same as for (a), but representing trace \#9.

To elaborate on some further details of the model computations: Figures $5 \mathrm{a}$ and $5 \mathrm{~b}$ show more results from one of the runs, namely the one representing trace \#8. Figure 5a shows the computed meteor temperatures vs altitude, showing the successive evaporation of $\mathrm{MgO}, \mathrm{FeO}$ and $\mathrm{SiO}_{2}$; and Figure $5 \mathrm{~b}$ shows the computed variations of the effective ablation coefficient $\sigma$ with altitude, including the total $\sigma$ and the separate evaporative contribution.

For meteors composed of mixtures of materials the total vapor pressure at any point is the sum of the vapor pressures of the individual constituents, irrespective of their relative amounts. Then the evaporation rate for each component should be given by the Langmuir equation (Eq 7), irrespective of the fraction of that component in the mixture. Then at each instant all of the constituents will be 
evaporating simultaneously at rates proportional to their individual vapor pressures - until such times as each successive constituent disappears by evaporation. One result of this is that the meteor temperature rises in a series of discrete steps, where the steps correspond to the disappearances of successive components. This is illustrated in Figure 5a. The ablation coefficients also exhibit a stepwise character, but with sharp decreases between successive steps, as is shown in Figure 5b. It is notable of course that the ablation coefficients are by no means constant, in contradiction to the assumption in Eq 5 .

Our assumed constant sputtering contribution to the ablation coefficient produces a substantial difference in the computed ablation and deceleration rates. Figure 5c shows the computed ablation coefficient vs altitude for the same case as that shown in Figures $5 \mathrm{a}$ and $5 \mathrm{~b}$, where in the computation the sputtering energy $\mathrm{E}^{*}$ was raised to $1000 \mathrm{eV}$ per molecule, so that the sputtering contribution to sigma was reduced to $3.1 \times 10^{-14} \mathrm{~s}^{2} / \mathrm{cm}^{2}$, which would be in better agreement with the laboratory data. The result was a considerable reduction in the effective average ablation coefficients and a reduction in the meteor deceleration rates.
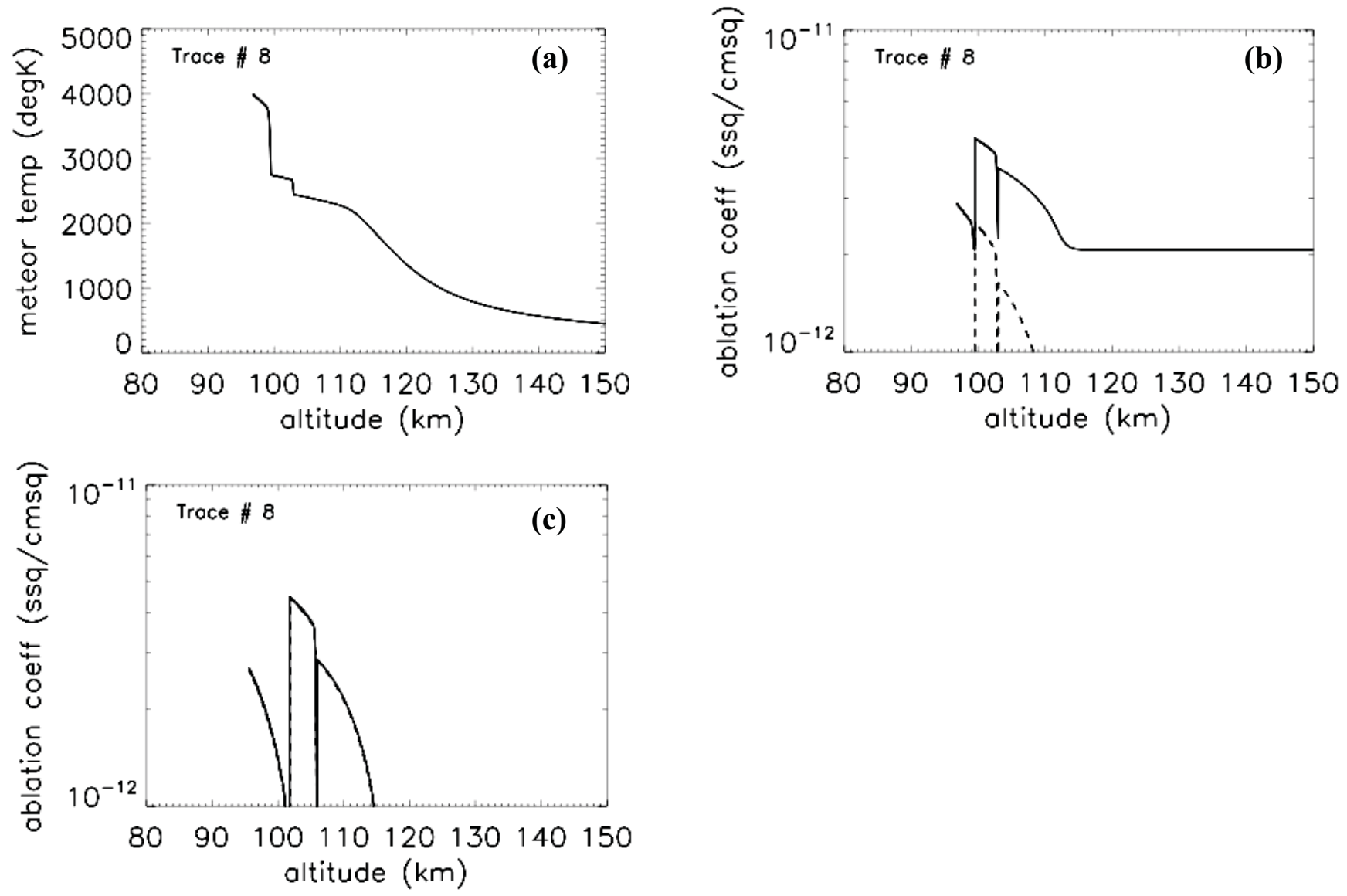

Figures 5. (a) Computed temperature history for the same meteor as in 3a. (b) Computed ablation coefficient vs altitude for the same meteor as in 3a, and 5a. The dashed curve is the evaporative contribution, and the solid curve is the total. (c) Computed ablation coefficient vs altitude for the same case as in Figures 5a,b, when in the computation the assumed sputtering energy $\mathrm{E}^{*}$ is raised to $1000 \mathrm{eV}$. 


\section{Direct Determination of $\rho_{\mathrm{s}}{ }^{2}$ from the Deceleration Data}

The quantity $m \rho_{\mathrm{s}}{ }^{2}$ can be determined directly from the fitted velocity and deceleration rate data without the need to use the computer model, but assuming only that the meteor is a sphere. Then the rate of deceleration is as given by Eq 3. For a sphere of density $\rho_{\mathrm{s}}$ the quantity $\pi \mathrm{r}^{2}$ is $\pi \mathrm{r}^{2}=1.209\left(\mathrm{~m} / \mathrm{\rho}_{\mathrm{s}}\right)^{2 / 3}$. Then, combining these two equations we obtain

$$
m \rho_{\mathrm{s}}^{2}=\left[-1.209 \rho \mathrm{v}^{2} /(\mathrm{dv} / \mathrm{dt})\right]^{3}
$$

The values of $m \rho_{\mathrm{s}}{ }^{2}$ thus determined are of course very sensitive to errors in the measured/fitted deceleration rates. If we nevertheless proceed to evaluate the $m \rho_{\mathrm{s}}{ }^{2}$ from the data fits for 27 of the measured traces, and plot them as functions of altitude, the result is Figure 6. Only about twenty of these curves seem to be believable, namely those that slope upward to the right and are concave downward. This set of twenty is the same as the twenty for which we found agreement between the computed and measured deceleration rates as described in the previous section.

Despite the expected inaccuracies in these $m \rho_{\mathrm{s}}{ }^{2}$ values, it is of interest to compare them with the corresponding values that we determined in the previous section from fitting the model-computed decelerations to the data. Table 2 shows, for each of the twenty chosen traces, (1) the initial (uppermost) altitude, (2) the initial value of $\mathrm{ms}_{\mathrm{s}}{ }^{2}$ at that altitude, as determined directly from the data using Eq 11, (3) the $m \rho_{\mathrm{s}}{ }^{2}$ value at the same altitude as computed with the model, and (4) the value extrapolated to the top of the atmosphere using the model. As expected, the agreement between the values in columns 3 and 4 is not very good, but nor is it extremely bad in most cases. The worst disagreement is for traces 4 and 27, which are also exceptional in that their altitudes are more than fifteen kilometers higher than the rest.

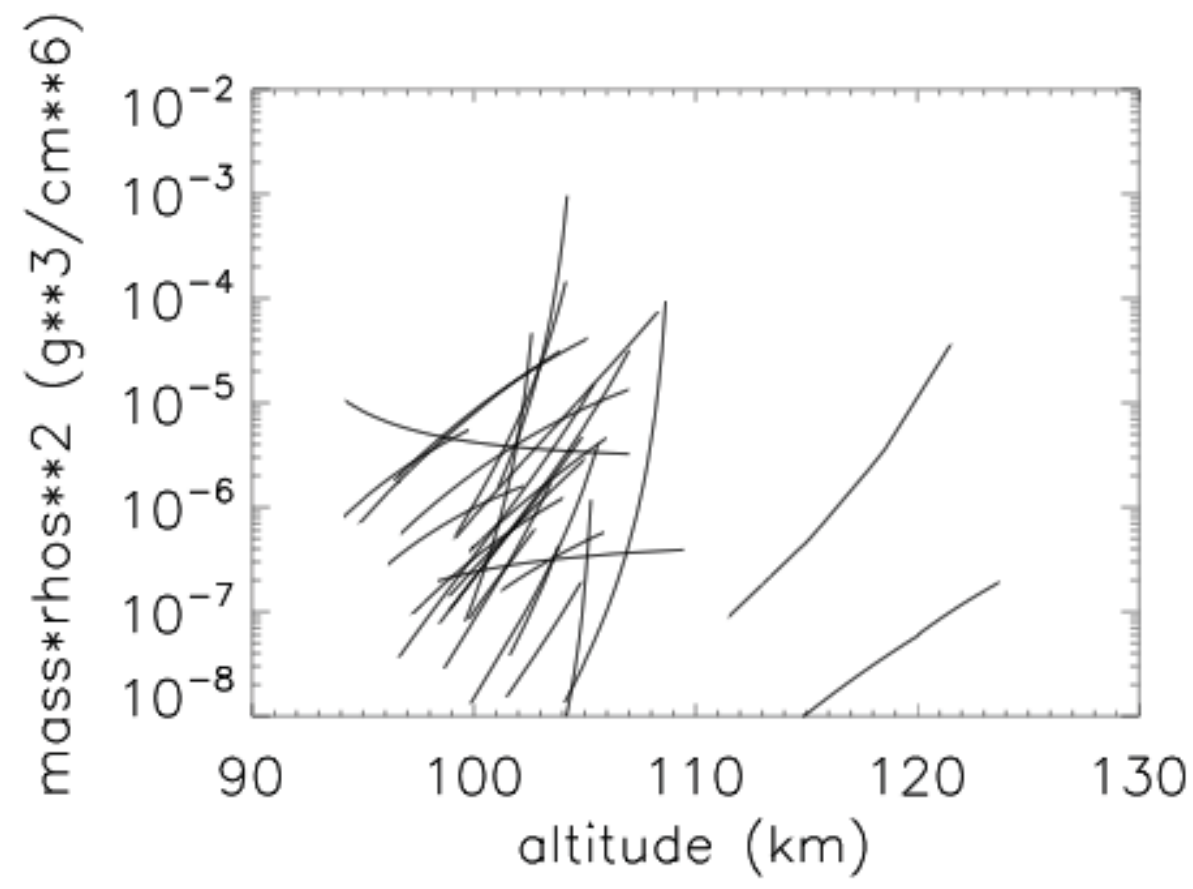

Figure 6. A composite plot of the combined variable $\mathrm{m} \rho_{\mathrm{s}}{ }^{2}$ for 27 traces. 
Table 2. Comparisons between values of $\mathrm{m}_{\mathrm{s}}{ }^{2}$ determined directly from the data via Eq 11 and values determined by the method described in Section III.

\begin{tabular}{|l|l|l|l|l|}
\hline Trace \# & $\begin{array}{l}\text { Initial altitude } \\
(\mathrm{z} 1) \text { (kilometers) }\end{array}$ & $\begin{array}{l}\mathrm{m}_{\mathrm{s}}{ }^{2} \text { at } \mathrm{z} 1 \\
\text { from Eq } 11\end{array}$ & $\begin{array}{l}\mathrm{m} \mathrm{s}_{\mathrm{s}}{ }^{\mathrm{at}} \mathrm{z} 1 \\
\text { from model }\end{array}$ & $\begin{array}{l}\left.\mathrm{m} \mathrm{s}_{\mathrm{s}}{ }^{\mathrm{z}}=\infty\right) \\
\text { from model }\end{array}$ \\
\hline 1 & 104 & $2 . \mathrm{e}-6$ & $1.6 \mathrm{e}-6$ & $8.4 \mathrm{e}-6$ \\
\hline 4 & 124 & $2 . \mathrm{e}-7$ & $3.0 \mathrm{e}-8$ & $4.6 \mathrm{e}-8$ \\
\hline 8 & 105 & $3 . \mathrm{e}-5$ & $8.5 \mathrm{e}-5$ & $1.5 \mathrm{e}-4$ \\
\hline 9 & 108 & $1 . \mathrm{e}-4$ & $1.4 \mathrm{e}-5$ & $2.0 \mathrm{e}-5$ \\
\hline 10 & 107 & $3 . \mathrm{e}-5$ & $6 . \mathrm{e}-6$ & $1.0 \mathrm{e}-5$ \\
\hline 11 & 102 & $1.5 \mathrm{e}-6$ & $4.5 \mathrm{e}-6$ & $7 . \mathrm{e}-5$ \\
\hline 12 & 104 & $2 . \mathrm{e}-5$ & $2.1 \mathrm{e}-5$ & $3.2 \mathrm{e}-5$ \\
\hline 13 & 104 & $1 . \mathrm{e}-6$ & $4.0 \mathrm{e}-6$ & $1.2 \mathrm{e}-5$ \\
\hline 15 & 105 & $3 . \mathrm{e}-6$ & $4.1 \mathrm{e}-6$ & $1.2 \mathrm{e}-5$ \\
\hline 16 & 103 & $4 . \mathrm{e}-7$ & $1.4 \mathrm{e}-6$ & $1.6 \mathrm{e}-5$ \\
\hline 17 & 105 & $2 . \mathrm{e}-5$ & $6 . \mathrm{e}-6$ & $1.4 \mathrm{e}-5$ \\
\hline 20 & 104 & $3 . \mathrm{e}-7$ & $4.3 \mathrm{e}-7$ & $4.1 \mathrm{e}-6$ \\
\hline 23 & 105 & $2 . \mathrm{e}-7$ & $3 . \mathrm{e}-7$ & $3 . \mathrm{e}-6$ \\
\hline 24 & 106 & $3 . \mathrm{e}-6$ & $1.0 \mathrm{e}-6$ & $4.2 \mathrm{e}-6$ \\
\hline 25 & 107 & $1 . \mathrm{e}-5$ & $2.1 \mathrm{e}-5$ & $3.4 \mathrm{e}-5$ \\
\hline 27 & 123.5 & $3 . \mathrm{e}-5$ & $2.0 \mathrm{e}-7$ & $2.7 \mathrm{e}-7$ \\
\hline 28 & 106 & $4 . \mathrm{e}-6$ & $7 . \mathrm{e}-6$ & $1.5 \mathrm{e}-5$ \\
\hline 29 & 106 & $4 . \mathrm{e}-7$ & $1.1 \mathrm{e}-6$ & $3.1 \mathrm{e}-6$ \\
\hline 30 & 105 & $4 . \mathrm{e}-6$ & $2.7 \mathrm{e}-6$ & $6.8 \mathrm{e}-6$ \\
\hline 32 & 99.5 & $4 . \mathrm{e}-6$ & $1.3 \mathrm{e}-5$ & $8.2 \mathrm{e}-5$ \\
\hline
\end{tabular}

This procedure (i.e. using Eq 11) has the obvious advantage that it does not use any assumptions about the meteor composition, whereas in using the model a composition must be assumed. In both cases we assume a spherical meteor shape. Using the model has the advantage that it allows us to extrapolate the $m \rho_{\mathrm{s}}{ }^{2}$ to the top of the atmosphere.

\section{Discussion}

With the present 32-trace data set the velocities and trajectory inclinations were arrived at by differencing the measured $3 \mathrm{D}$ position vs time data. In view of the expected errors inherent to numerical differencing procedures, it has been encouraging to find that these $3 \mathrm{D}$ velocity data can be fitted so well as quadratic functions of Q. However, it is also not surprising to find that when we try to infer the $m \rho_{\mathrm{s}}{ }^{2}$ quantities directly from these data, as in the previous section, that many of the $m \rho_{\mathrm{s}}{ }^{2} \mathrm{vs}$ altitude curves look crazy. We are currently in the process of analyzing a much larger set of ALTAIR meteor data from 2007-2008, where it appears to be possible to obtain more accurate velocities from range Doppler measurements. We are hopeful that when these data are available we can go through these same procedures to obtain a larger set of more reliable $m \rho_{\mathrm{s}}{ }^{2}$ values. With such a data set we will be able to extract more detailed information about the evaporation rates, ablation coefficients etc.

For purposes of evaluating the initial values of $m \rho_{\mathrm{s}}{ }^{2}$ we have chosen to use the computer model to find the values that produce the best fits to the deceleration rate data. However, a serious problem 
with that is that the model results are sensitive to the assumed chemical compositions of the meteors, which are of course not known. Our assumption of the olivine-like composition was convenient because the necessary data on vapor pressures and heats of sublimation of the decomposition products were available in the literature.

In the process of comparing the model results to the deceleration data we found that the fits were improved when we assumed a rather large sputtering contribution to the effective ablation coefficients, namely $2 \times 10^{-12} \mathrm{~s}^{2} / \mathrm{cm}^{2}$. This value is significantly larger than values that have been determined in laboratory measurements of sputtering from energetic ion bombardment of solid target materials (Behrisch 1981, Bodhansky et al 1980, Lebedinets and Shushkova 1970, Ratcliff et al 1997, Tielens et al 1994)). However, with meteors entering the atmosphere the collision fluxes are much larger than in the laboratory experiments, and for most of the time the meteors are molten. Then the laboratory results may not be directly comparable

The model results show that the meteor temperatures almost invariably exceed the melting points before very much ablation occurs. Nevertheless, in our twenty selected cases the ablation and deceleration rates appear to vary smoothly, without obvious evidence of fragmentation. This seems quite surprising. However, in the remaining twelve cases the failure to fit our model could be an indication of fragmentation.

In our computer model we have assumed that the vapors emitted by the meteors are molecular rather than atomic. This seems to differ from the assumptions in the model described by Vondrak et al 2008, and Janches et al 2009. In view of the fact that the dissociation energies of, for instance, $\mathrm{SiO}_{2}$, $\mathrm{MgO}$ and $\mathrm{FeO}$ are very much larger than their sublimation energies, it seems unlikely that the evaporation products would be atomic. On the other hand, subsequent collisions of the evaporated molecules with background air molecules would certainly lead to dissociation and/or ionization.

\section{Summary}

It appears that with most of these 32 radar traces the range and altitude vs time measurements are of sufficient quality to allow us to extract reliable velocities, trajectory inclinations and deceleration rates. In about $80 \%$ of the cases the velocities can be fitted with good accuracy as quadratic functions of the integrals of the air densities along the measured trajectories, and the time derivatives of these functions provide reasonable values of deceleration rates. We have used these fitted velocities and deceleration rates together with a computer model to determine best-fit values of the quantity $m \rho_{\mathrm{s}}{ }^{2}$, the product of the initial meteor mass times its mass density squared, successfully in 20 of the 32 cases. The model, which we have described, treats the coupled processes of meteor deceleration through air molecule collisions and the associated heating of the meteor, together with cooling by blackbody emission and by evaporation of its constituents, and the rate of loss of mass through evaporation and by sputtering. This procedure does not provide information about the separate quantities $m$ and $\rho_{\mathrm{s}}$. However, separate estimates of the masses $\mathrm{m}$ have been obtained from the measured radar scattering cross sections, using an electrostatic scattering model. By combining these $m$ values with the $m \rho_{\mathrm{s}}{ }^{2}$ we have obtained values of $\rho_{\mathrm{s}}$, almost all of which fall in the range between 0.1 to $1 \mathrm{~g} / \mathrm{cm}^{3}$. We have also described a process by which we can obtain $m \rho_{\mathrm{s}}{ }^{2}$ values directly from the velocity and deceleration data without using the computer model, although the results are very sensitive to errors in the decelerations. 